\title{
Significant increase in non-COVID-19 related ophthalmology publications during the COVID-19 era: is this a new normal?
}

\author{
Jeremy Reitinger $\mathbb{I}^{1} \cdot$ Samiksha Fouzdar Jain ${ }^{2} \cdot$ Donny Suh ${ }^{2}$
}

Received: 20 September 2020 / Revised: 28 September 2020 / Accepted: 2 October 2020 / Published online: 13 October 2020

(c) The Royal College of Ophthalmologists 2020

COVID-19 has affected nearly every aspect of society, with expansive changes on everything from the economy [1] to mental health [2]. These changes have been particularly noticeable within the field of medical literature, with an unprecedented surge in COVID-19 related publications within numerous specialties [3] and a significant decrease in acceptance time of COVID-19 publications approaching 6 days [4]. Due to the current changing landscape within medical literature and limited knowledge of the effect of these changes on ophthalmology literature, we sought to examine the effects of COVID-19 on ophthalmology publication trends.

We examined the amount of publications within PubMed each month from January 2020 to August 2020 using the keywords "ophthalmology" and "COVID-19." In addition, we examined new clinical trials within multiple databases (Clinicaltrials.gov, University Hospital Medical Information Network, Australian New Zealand Clinical Trials Registry, and International Standard Randomized Controlled Trial Number Registry) using the keywords "ophthalmology" or "eye diseases." We calculated a yearly growth rate using the sum of all publications throughout an entire year dating back to 2016, not accounting for monthly variation. The amount of monthly publications in 2020 were compared to the equivalent month in 2019.

As compared to 2019, there has been a $22.1 \%$ increase in ophthalmology publications (2838) from March through August of 2020, most prominent from May through August (increase of $28.8 \%, 2222$ publications) (Table 1). It is interesting to note that only $3.9 \%$ of ophthalmology publications $(742 / 18,817)$ from January through August 2020

Jeremy Reitinger

jeremy.reitinger@yahoo.com

Creighton University School of Medicine, Omaha, NE, USA

2 Department of Pediatric Ophthalmology \& Adult Strabismus, Children's Hospital and Medical Center, Omaha, NE, USA were related to COVID-19 (Table 1). After adjustment for COVID-19 related publications and a 5.3\% average annual growth rate since 2016 (Table 2), we found a $9.4 \%$ increase of publications (1508) from March to August of 2020 not attributed to a specific factor.

The significantly higher rate of total publications in ophthalmology during 2020 is likely attributable to a decrease in clinical workload and more time available for research as well as academic activities. This has occurred when communities have been placed under lockdown or clinics have decreased volume to accommodate the changing COVID-19 climate. This rise in publications is only partly due to a surge in COVID-19 publications. The growth of research during this time, whether COVID-19 related or not, does pose the risk for publication of undervetted information or opinion pieces without solid evidence $[4,5]$. Research has always been a meticulous and gradual process, but the demand for rapid answers to adjust global policy has changed the research community fundamentally [6]. Although many peer-reviewed journals have accelerated publication of COVID-19 related studies by increasing the number of reviewers and preprints [7], journals may be aware there is an increase in non-COVID-19 related publications as well.

In addition, there was no significant change in ophthalmology specific registered clinical trials during this time frame in any clinical trial database. We hypothesize that the changing clinical climate has made new clinical trials difficult to begin and therefore researchers have not been able to begin new clinical trials. This could be due to a decrease in research funding as more funding is put towards researching COVID-19. Additionally, COVID-19 related restrictions on facility usage and personnel access may limit clinical trials.

It is difficult to determine if this increase in research publications will continue, or if the trend will correct itself as clinical work returns to pre-COVID workflows. 
Table 1 Monthly ophthalmology publication totals in 2019 and 2020.

$20192020 \quad$ Increase COVID-19
from 2019 related, 2020 to $2020(\%)$ (\% monthly total)

\begin{tabular}{lllrr}
\hline January & 2758 & 2468 & -10.5 & $13(0.5 \%)$ \\
February & 1773 & 1976 & 12.7 & $6(0.3 \%)$ \\
March & 1870 & 2167 & 15.8 & $23(1.1 \%)$ \\
April & 1950 & 2269 & 16.4 & $75(3.3 \%)$ \\
May & 1962 & 2382 & 21.4 & $141(5.9 \%)$ \\
June & 1847 & 2401 & 30.0 & $141(5.9 \%)$ \\
July & 1997 & 2721 & 36.3 & $195(7.2 \%)$ \\
August & 1909 & 2433 & 27.5 & $148(6.1 \%)$ \\
Total, & 16,046 & 18,817 & 17.3 & 742 \\
January-August & & & & \\
\hline
\end{tabular}

Table 2 Yearly publication totals in ophthalmology.

\begin{tabular}{lllll}
\hline & 2016 & 2017 & 2018 & 2019 \\
\hline Publications & 16,904 & 17,884 & 18,823 & 19,721 \\
\% increase from previous year & & $5.8 \%$ & $5.3 \%$ & $4.8 \%$ \\
\hline
\end{tabular}

\section{Compliance with ethical standards}

Conflict of interest The authors declare that they have no conflict of interest.
Publisher's note Springer Nature remains neutral with regard to jurisdictional claims in published maps and institutional affiliations.

\section{References}

1. Nicola M, Alsafi Z, Sohrabi C, Kerwan A, Al-Jabir A, Losifidis C, et al. The socio-economic implications of the coronavirus pandemic (COVID-19): a review. Int J Surg. 2020;78:185-93.

2. Shah K, Kamrai D, Mekala H, Mann B, Desai K, Patel RS. Focus on mental health during the coronavirus (COVID-19) pandemic: applying learnings from the past outbreaks. Cureus. 2020;12:e7405.

3. Kambhampati SBS, Vaishya R, Vaish A. Unprecedented surge in publications related to COVID-19 in the first three months of pandemic: a bibliometric analytic report. J Clin Orthop Trauma. 2020;11:S304-S6.

4. Palayew A, Norgaard O, Safreed-Harmon K, Andersen TH, Rasmussen LN, Lazarus JV. Pandemic publishing poses a new COVID-19 challenge. Nat Hum Behav. 2020;4:666-9.

5. Bagdasarian N, Cross GB, Fisher D. Rapid publications risk the integrity of science in the era of COVID-19. BMC Med. 2020;18:192.

6. Ranganathan S. "Slow research" in the time of Covid-19. IJME. 2020;05:212-4.

7. Callaway E. Will the pandemic permanently alter scientific publishing? Nature 2020;582:167-8. 